\title{
Hadron Electromagnetic Form Factors In Time-Like Region
}

\author{
Alaa Dbeyssi ${ }^{*+}$ \\ CNRS/IN2P3, Institut de Physique Nucléaire, UMR 8608, 91405 Orsay, France \\ E-mail: dbeyssi@ipno.in2p3.fr
}

\begin{abstract}
Electromagnetic form factors (FFs) of hadrons are fundamental quantities which describe the structure and the internal dynamics of these system. Elastic electron scattering on hadrons allows to access electromagnetic FFs in the space-like (SL) region. In time-like (TL) region, FFs are complex and can be measured through annihilation reactions. Crossing symmetry and analyticity connect the two kinematical regions.

At the future accelerator complex FAIR, in Darmstadt, the PANDA (antiProton ANnihilation at DArmstadt) collaboration plans to measure proton time-like FFs using an antiproton beam of momentum up to $15 \mathrm{GeV} / \mathrm{c}$ and luminosity $\mathscr{L}=2 \cdot 10^{32} \mathrm{~cm}^{-2} \mathrm{~s}^{-1}$.

The Vector Meson Dominance (VMD) models describe space-like FFs, where most of the data exist, and have the necessary analytical properties to be extended into the TL region. We discuss predictions for the reactions $\bar{p}+p \leftrightarrow e^{+}+e-$ in the kinematic regime accessible at PANDA.

We present an extension of a VMD parametrization to spin one hadrons ( $\rho$-meson). As no data exist, the parameters are adjusted to reproduce theoretical predictions in the SL region. The parametrization is analytically extended to the TL region including the necessary imaginary component of FFs, through the resonance widths. A correspondence between the formalism and the helicity amplitudes is established in order to analyse the constraint on the TL $\rho$-meson FFs measured by the BaBar collaboration for the reaction $e^{+}+e^{-} \rightarrow \bar{\rho}+\rho$.
\end{abstract}

Xth Quark Confinement and the Hadron Spectrum,

October 8-12, 2012

TUM Campus Garching, Munich, Germany

\footnotetext{
* On the behalf of the PANDA collaboration.

${ }^{\dagger}$ Boursier du CNRS libanais/LNCSR Scholar.
} 


\section{Introduction}

The understanding of the internal structure of hadrons has been an object of extended experimental and theoretical studies for many decades. The elastic scattering of electrons by hadrons, was an effective tool to investigate their electromagnetic structure. Assuming $\mathrm{C}$ and $\mathrm{P}$ invariance of hadron electromagnetic interactions, a particle with spin $S$ is described by $2 S+1$ independent electromagnetic FFs. Protons and neutrons are described by two FFs, electric, $G_{E}$, and magnetic, $G_{M}$. A deuteron (spin one particle) is described by three FFs, charge $G_{C}$, magnetic $G_{M}$, quadrupole $G_{Q}$. In Born approximation, where the interaction between electrons and hadrons occurs through the exchange of one virtual photon, FFs are analytical functions of one kinematical variable, $q^{2}$ (mass of the virtual photon), which parametrizes the internal size inside the hadron. The elastic FFs are real in scattering processes (Space-Like (SL) region), and become complex in the Time-Like region accessible by the annihilation channels, $\bar{H}+H \leftrightarrow e^{+}+e^{-}$.

Due to the low luminosity achieved in the experiments, the existing data on the TL nucleon FFs were analysed under the assumption $\left|G_{E}\right|=\left|G_{M}\right|$ or $\left|G_{E}\right|=0$. No individual determination of the two FFs was done. The measurement of the proton FFs by the proton-antiproton annihilation into electromagnetic channels is one of the goals of future PANDA (Antiproton Annihilation at Darmstadt) experiment [1]. PANDA is a $\sim 4 \pi$ fixed target detector, designed to achieve momentum resolution at the percent level for charged particles, high rate capability up to $10 \mathrm{MHz}$ and good vertex resolution $(\sim 100 \mu \mathrm{m})$. Evaluations based on Monte Carlo simulations show that the individual determination for proton Time-Like FFs can be done up to to $q^{2} \sim 14(\mathrm{GeV} / \mathrm{c})^{2}$, and, with a precise knowledge of the luminosity, the absolute cross section can be measured up to $q^{2} \sim 28(\mathrm{GeV} / \mathrm{c})^{2}$ [2]. An improvement of at least one order of magnitude is expected, compared to the existing data.

Different models exist to describe the non-perturbative structure of hadrons. Models based on vector meson dominance, have been successful in describing the nucleon electromagnetic FFs in SL and TL regions [3]. We discuss in this note the extension of these models to spin-one hadrons as the $\rho$-meson.

\section{Spin one hadrons, VMD inspired model}

The deuteron is the spin one hadron which has been largely discussed in the literature. Data on the three SL deuteron FFs, using the ed elastic scattering, are available up to momentum transfer squared $Q^{2}=1.9 \mathrm{GeV}^{2}$. The measurement of deuteron FFs in the TL region is beyond the experimental possibilities, but information on the TL spin one hadrons can be extracted form the reaction $e^{+}+e^{-} \rightarrow \rho^{-}+\rho^{+}$.

The BaBar collaboration has detected four pions identifying the $e^{+}+e^{-} \rightarrow \rho^{+}+\rho^{-}$reaction [4]. The results were given in terms of helicity amplitudes. This unique experimental data point puts a constraint on the existent $\rho$-meson FFs parametrizations.

In Ref. [5], one can find a simple parametrization, based on VMD approach, for the electromagnetic FFs of the $\rho$-meson. The parametrization reproduced the predictions from Ref. [6] in SL region. The extension of the model to TL region was made by analytical continuation, introducing an imaginary part through widths for the effective particles carrying the electromagnetic 


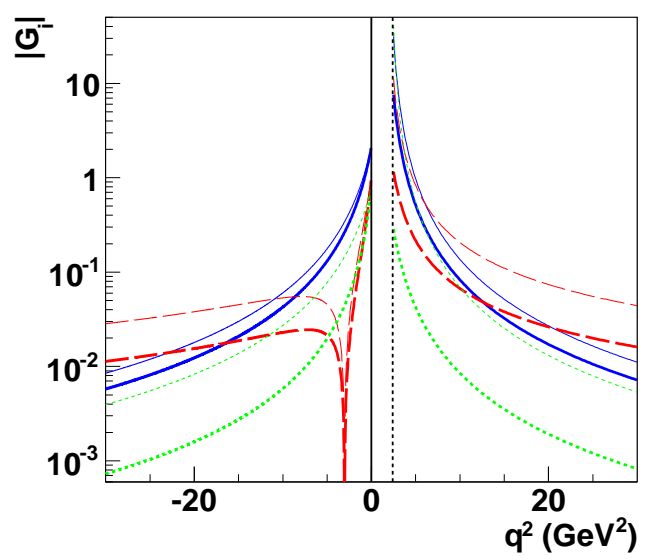

Figure 1: Absolute value of $\rho$-meson FFs $\left|G_{i}\right|, i=C$ (red, dashed line), $Q$ (green, dotted line), $M$ (blue, solid line), from Ref. [7] (thick lines) and from Ref. [5] (thin lines) as functions of $q^{2}$ in space and time-like regions. The black, dotted line indicates the kinematical threshold for the considered reaction.

interaction. Keeping the same form of parametrization, a small readjustment of the mass parameters (mass of the effective particles) allows to correctly reproduce the helicity amplitudes measured in the BaBar experiment [7]. The difference between the old and the present parametrization due to the experimental constraint is smaller than an order of magnitude as illustrated in Fig. 1.

\section{Conclusion}

Models based on VMD give useful parametrization for electromagnetic FFs of spin $1 / 2$ and spin one hadron in SL and TL regions. We showed that the VMD parametrization can reproduce the unique measurement on the spin one TL FFs given in terms of helicity amplitudes. Future experiments as the PANDA experiment will provide a powerful test of the existent models of the nucleon FFs in a wide $q^{2}$ range.

\section{References}

[1] The PANDA Collaboration, Physics Performance Report for PANDA: Strong Interaction Studies with Antiprotons, arXiv:0903.3905 [hep-ex].

[2] M. Sudol, M. C. Mora Espi, E. Becheva, J. Boucher, T. Hennino, R. Kunne, D. Marchand and S. Ong et al., Eur. Phys. J. A 44, 373 (2010) [arXiv:0907.4478 [nucl-ex]].

[3] F. Iachello, A. Jackson, and A. Lande, Phys.Lett. B43, 191-196 (1973).

[4] B. Aubert, et al., Phys.Rev. D78, 071103 (2008).

[5] C. Adamuscin, G. Gakh, and E. Tomasi-Gustafsson, Phys.Rev. C75, 065202 (2007).

[6] J. de Melo, and T. Frederico, Phys.Rev. C55, 2043 (1997).

[7] A. Dbeyssi, E. Tomasi-Gustafsson, G. Gakh, and C. Adamuscin, Phys.Rev. C85, 048201 (2012). 